\section{A) Check for updates}

Cite this: Nanoscale, 2021, 13, 10829

\title{
Quantifying thermal transport in buried semiconductor nanostructures via cross-sectional scanning thermal microscopy $\dagger$
}

\author{
Jean Spièce, ${ }^{* a}$ Charalambos Evangeli, (iD † $^{a}$ Alexander J. Robson, ${ }^{a}$ \\ Alexandros El Sachat, ${ }^{b}$ Linda Haenel, ${ }^{e}$ M. Isabel Alonso, (D) ${ }^{c}$ Miquel Garriga, (D) ${ }^{c}$ \\ Benjamin J. Robinson, (D) a,d Michael Oehme, (D) ${ }^{\text {e }}$ Jörg Schulze, ${ }^{\text {e }}$ Francesc Alzina, ${ }^{b}$ \\ Clivia Sotomayor Torres ${ }^{\mathrm{b}, \mathrm{f}}$ and Oleg V. Kolosov (D) *a,d
}

\begin{abstract}
Managing thermal transport in nanostructures became a major challenge in the development of active microelectronic, optoelectronic and thermoelectric devices, stalling the famous Moore's law of clock speed increase of microprocessors for more than a decade. To find the solution to this and linked problems, one needs to quantify the ability of these nanostructures to conduct heat with adequate precision, nanoscale resolution, and, essentially, for the internal layers buried in the 3D structure of modern semiconductor devices. Existing thermoreflectance measurements and "hot wire" $3 \omega$ methods cannot be effectively used at lateral dimensions of a layer below a micrometre; moreover, they are sensitive mainly to the surface layers of a relatively high thickness of above $100 \mathrm{~nm}$. Scanning thermal microscopy (SThM), while providing the required lateral resolution, provides mainly qualitative data of the layer conductance due to undefined tip-surface and interlayer contact resistances. In this study, we used cross-sectional SThM (xSThM), a new method combining scanning probe microscopy compatible Ar-ion beam exit nanocross-sectioning (BEXP) and SThM, to quantify thermal conductance in complex multilayer nanostructures and to measure local thermal conductivity of oxide and semiconductor materials, such as $\mathrm{SiO}_{2}$, $\mathrm{SiGe}_{x}$ and $\mathrm{GeSn}_{y}$. By using the new method that provides $10 \mathrm{~nm}$ thickness and few tens of nm lateral resolution, we pinpoint crystalline defects in SiGe/GeSn optoelectronic materials by measuring nanoscale thermal transport and quantifying thermal conductivity and interfacial thermal resistance in thin spin-on materials used in extreme ultraviolet lithography (eUV) fabrication processing. The new capability of xSThM demonstrated here for the first time is poised to provide vital insights into thermal transport in advanced nanoscale materials and devices.
\end{abstract}

Received 10th December 2020, Accepted 13th May 2021

DOI: $10.1039 /$ dOnr08768h

rsc.li/nanoscale

\section{Introduction}

Nanomanufacturing that became a major foundation for modern technological development directly relies on the rapid and versatile quantitative characterization of devices on the

\footnotetext{
${ }^{a}$ Physics Department, Lancaster University, LA1 4YB, UK.

E-mail: o.kolosov@lancaster.ac.uk, spiece.jean@gmail.com

${ }^{b}$ Catalan Institute of Nanoscience and Nanotechnology (ICN2), CSIC and BIST, Campus UAB, Bellaterra, 08193 Barcelona, Spain

${ }^{c}$ Institut de Ciència de Materials de Barcelona, ICMAB-CSIC, Campus UAB, 08193 Bellaterra, Spain

${ }^{d}$ Material Science Institute, Lancaster University, Lancaster, LA1 4YB, UK

${ }^{e}$ University of Stuttgart, Pfaffenwaldring 47, 70569 Stuttgart, Germany

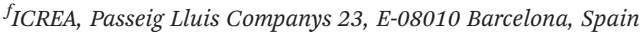

$\dagger$ Electronic supplementary information (ESI) available. See DOI: 10.1039/ d0nr08768h

\$Current address: Department of Materials, University of Oxford, Oxford, OX1 3PH, UK.
}

nanoscale. While scanning and transmission electron microscopies (SEM and TEM) provide excellent nanostructural characterization, the means for the mapping of materials and devices specifically physical properties are lagging well behind. In particular, one of the most vital characteristics of materials at the nanoscale, their ability to transfer or impede heat, is also one of the most difficult to characterize. The microelectronic industry is struggling to dissipate heat generated by nanoscale hot spots in computer processor chips; ${ }^{1,2}$ the new nanostructured thermoelectrics rely on suppressing the detrimental thermal conductance pathways, the phase change memory that strives to replace both flash and dynamic memory need improved management of local heat generation to become a feasible alternative. ${ }^{3}$

However, the measurement of thermal conductivity, even in a simple geometry, such as a thin film on a substrate, presents significant challenges to traditional techniques, if the layer thickness is smaller than $100 \mathrm{~nm} .^{4}$ In particular, decoupling 
the thermal conductivity and the interfacial resistance between the film and the substrate, and accessing the in-plane thermal conductivity is difficult, and often not possible. ${ }^{5}$ Furthermore, as nanostructured device architectures are becoming more complex with increased layer number and innovative threedimensional (3D) geometries, such as FIN-FET transistors and low- $k$ interconnects, new approaches are required to probe the thermal transport in buried layers and the interlayer interfaces. Existing techniques are mostly limited to either surface or bulk probing, and cannot assess thermal transport in buried nanostructures.

Scanning probe microscopy (SPM)-based techniques, such as scanning thermal microscopy (SThM), can provide an efficient solution with lateral resolution on the order of a few nanometers to a few tens of nanometers. ${ }^{6,7}$ SThM uses a probe with a heated thermal sensor and a nanoscale sharp apex that is brought in the thermal contact with the sample, and scanned in a raster pattern over the surface of the probed sample. The electrical resistance of the probe sensor is proportional to its temperature, and is monitored during scanning. By measuring the probe temperature, heat transfer properties of the sample can be deduced. ${ }^{8-10}$ However, whilst using SThM to quantify the overall thermal conductance of the complex 3D structure remains challenging but possible, assessing thermal conductivities of the individual structure elements buried in the 3D device and reliably separating them from the interfacial thermal resistance remains out-of-reach of the technique. Several groups ${ }^{11-16}$ devoted their studies to temperature and conductance measurements using SThM. While Park et $a .^{13}$ reported measurements of ErAs/GaAs MBE superlattices with $6 \mathrm{~nm}$ RMS roughness, Juszczyk et al. ${ }^{14}$ used craters in photonic structures to access subsurface materials. If the structure allows a cleavage, such as in coherent crystalline materials, these can be probed as demonstrated by Jung et al. ${ }^{15}$ where an LED cleavage was used to map the nanoscale temperature distribution during its operation. However, all the methods reported the lack of reproducibility, can only be used to study a small set of structures, and most prominently use ill-defined surfaces, which creates major hurdles for the SThM probe measurements.

To address these challenges, here, we demonstrate crosssectional SThM (xSThM), a new method, which combines SThM with beam-exit nano-cross-sectional polishing (BEXP), a nano-cross-sectioning tool, that creates an easily accessible close to atomically flat section through a 3D structure enabling the SPM analysis of the subsurface layers of the studied material or device. ${ }^{17,18}$ The cross-sectioned surface has a wedge-like geometry and sub-nm surface roughness and is fully compatible with SThM enabling thermal transport measurements as a function of material thickness, which changes depending on the position of the probe across the cut.

We demonstrate the capabilities of this new method by exploring the heat transport in the complex buried semiconductor and optoelectronic nanostructures, quantifying the nanoscale gradients in composition, and revealing dis- locations and defects via variations in the local heat conductance. Furthermore, by analyzing the SThM signal of the wedge-shaped section, and applying an appropriate analytical model, we are able to independently extract the intrinsic thermal conductivity of isotropic material layers on a substrate. The ease of use of our approach and the extreme sensitivity to local physical properties renders it suitable for a broad range of samples and opens new paths for fundamental and applied research in nanomaterials and devices.

\section{Experimental}

\section{SThM compatible nano-cross-sectioning}

The nano-cross-sectioning (see Fig. 1a) described elsewhere $^{19,20}$ has been used to create an easily accessible surface section through a 3D structure for the subsurface SPM analysis of the material. ${ }^{17,18}$ Briefly, it uses three intersecting Ar-ion beams aligned to a single plane that impinge on a sample side at a small negative angle $\left(\sim-5^{\circ}\right)$ from below the sample surface. As the beam exits at a glancing angle to the sample surface, we call this technique beam-exit nano-crosssectional polishing. ${ }^{18,21}$ The cross-sectioned surface obtained has a wedge-like geometry and sub-nm surface roughness, making it fully compatible for studies via SPM methods. Equally essential, the glancing angle of the ion beam, and the

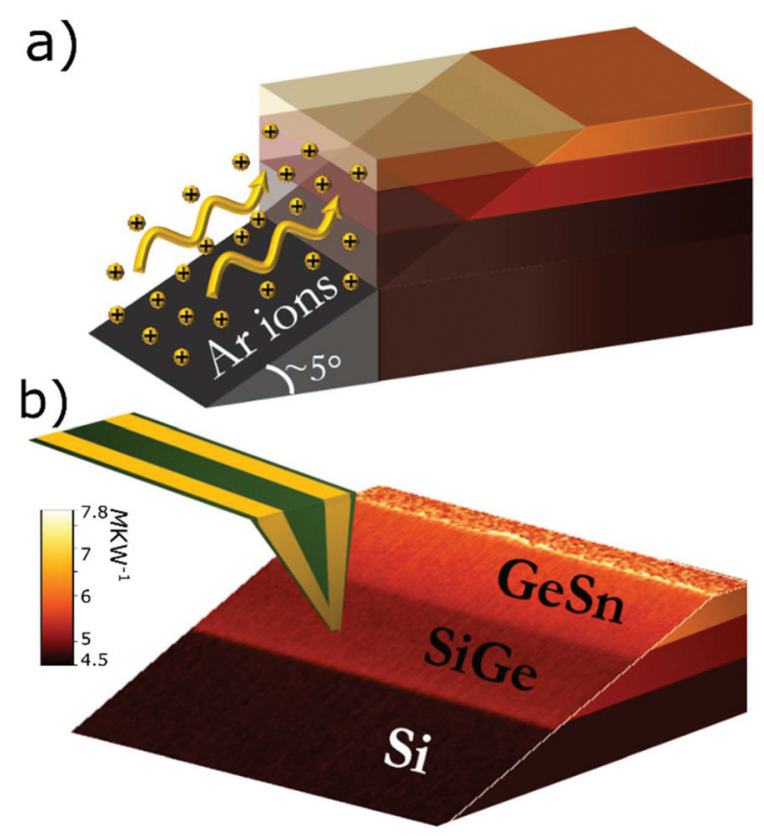

Fig. 1 (a) Schematic representation of nano-cross-sectioning beamexit cross-section polishing (BEXP); Ar ions impinge on the sample edge at a shallow negative angle $\left(\mathrm{ca} .-5^{\circ}\right)$ to its surface creating a SPM compatible cut adjacent to the intact sample surface. (b) Schematic representation of the XSThM measurement; SThM probe scans the cross-sectioned area of a multilayered material (The sample is presented in the next section). The 3D topography is overlaid with the SThM response. Image dimensions: $5 \times 5 \times 0.77 \mu \mathrm{m}$. 
inert nature of Ar results in negligible surface damage and practically no modification of the measured physical properties of the studied materials.

The cross-sectioned area was then thermally imaged via SThM (see Fig. 1b). Here, SThM measurements were performed in an ambient environment using a commercial SPM (Bruker MultiMode Nanoscope IIIa controller) and custom-built electronics. Acting as both a sensor and a heater, the SThM probe (Kelvin Nanotechnology, KNT-SThM-01a, 0.3 $\mathrm{N} \mathrm{m}^{-1}$ spring constant, $<100 \mathrm{~nm}$ tip radius) is based on a $\mathrm{SiN}$ cantilever with gold legs connecting to a Pd film evaporated on the tip. ${ }^{22}$ The SThM probe is one of the Wheatstone bridge resistors, thus allowing a precise monitoring of the probe resistance as explained elsewhere. ${ }^{7}$ In this study, we used an excess temperature of $50 \mathrm{~K}$ with respect to the environment. When scanning across the surface of the sample, the probe is biased by a combined $\mathrm{AC}+\mathrm{DC}$ voltage, and its resistance is monitored via a modified Wheatstone bridge. ${ }^{23}$ When the SThM probe at a temperature difference $\Delta T$ above the sample temperature contacts the sample surface, it cools down depending on the heat flux $q$ to the sample and, consequently, on the sample local heat transport characteristics. These temperature variations change the electrical resistance of the probe, which was quantified via calibration techniques described elsewhere, ${ }^{7,24}$ and used to determine the sample thermal properties. By measuring $q$ and $\Delta T$, the tipsample thermal contact resistance $R_{\mathrm{X}}=\Delta T / q$ can be found. To achieve this, we processed the acquired data using a calibration methodology that provides compensation for the tip geometry and ambient air conductance, ${ }^{25}$ and more importantly gives comparable quantitative measurements with the ones performed in a high vacuum environment (see ESI $\dagger$ for details).

\section{Results and discussion}

Measuring anisotropic thermal conductance on the nanoscale

In this section, we used the new method to investigate the nanoscale transport in complex anisotropic systems with the predominantly diffusive thermal transport, and qualitatively compare the thermal conductivities in such layers. Fig. 1b shows a 3D topography rendering section that is overlaid with color corresponding to the SThM output of an MBE grown multilayer sample of $\mathrm{Si} / \mathrm{Si}_{x} \mathrm{Ge}_{1-x} / \mathrm{Ge}_{\mathrm{Ge}} \mathrm{Sn}_{1-y}$. The GeSn materials represent a potential platform for Si manufacturingbased optoelectronics due to the possibility of achieving a direct bandgap. ${ }^{26-28}$ In this structure, first, a $100 \mathrm{~nm}$ Ge layer was grown on a silicon substrate. During the growth, Si atoms diffused inside the Ge layer at high process temperatures, ${ }^{29}$ and therefore, created an SiGe alloy of decreasing Si concentration, as the distance from the Si-Ge interface increased (see $\mathrm{ESI} \dagger$ for details). Then, another $100 \mathrm{~nm}$ Ge layer was grown creating a so-called Ge "virtual substrate". Finally, a $200 \mathrm{~nm}$ layer of $\mathrm{Ge}_{0.9} \mathrm{Sn}_{0.1}$ was grown on the top of this layer. The $z$-gradient in the $\mathrm{Si}_{x} \mathrm{Ge}_{1-x}$ and $\mathrm{Ge}_{y} \mathrm{Sn}_{1-y}$ layers brakes the isotropic nature of the sample, making it transversely isotropic. The three regions corresponding to the Si substrate, the Ge virtual substrate and the $\mathrm{Ge}_{0.9} \mathrm{Sn}_{0.1}$ layer were clearly observed in the thermal image. Fig. $2 \mathrm{~b}$ shows the dependence of $R_{\mathrm{X}}$ and the topography profile $x$ as a function of the height, $t$, of the sample nano-cross-section as obtained from a profile of the xSThM image (Fig. 2a) and the topography image, respectively. The height is quantified via topography since the thickness of the layer varies linearly with the position. ${ }^{21}$ The relatively low and spatially uniform thermal resistance in the Si substrate is adjacent to the steep resistance increase as the probe transited to the $\mathrm{Si}_{1-x} \mathrm{Ge}_{x}$ and Ge layers with a high density of misfit dislocations. This is followed by a decrease and roughly constant thermal resistance in what is believed to be a dislocation-free Ge layer. Finally, as the tip entered the $\mathrm{Ge}_{0.9} \mathrm{Sn}_{0.1}$ layer, the heat resistance increased again, continuing to increase towards the sample surface, suggesting an increase in the Sn

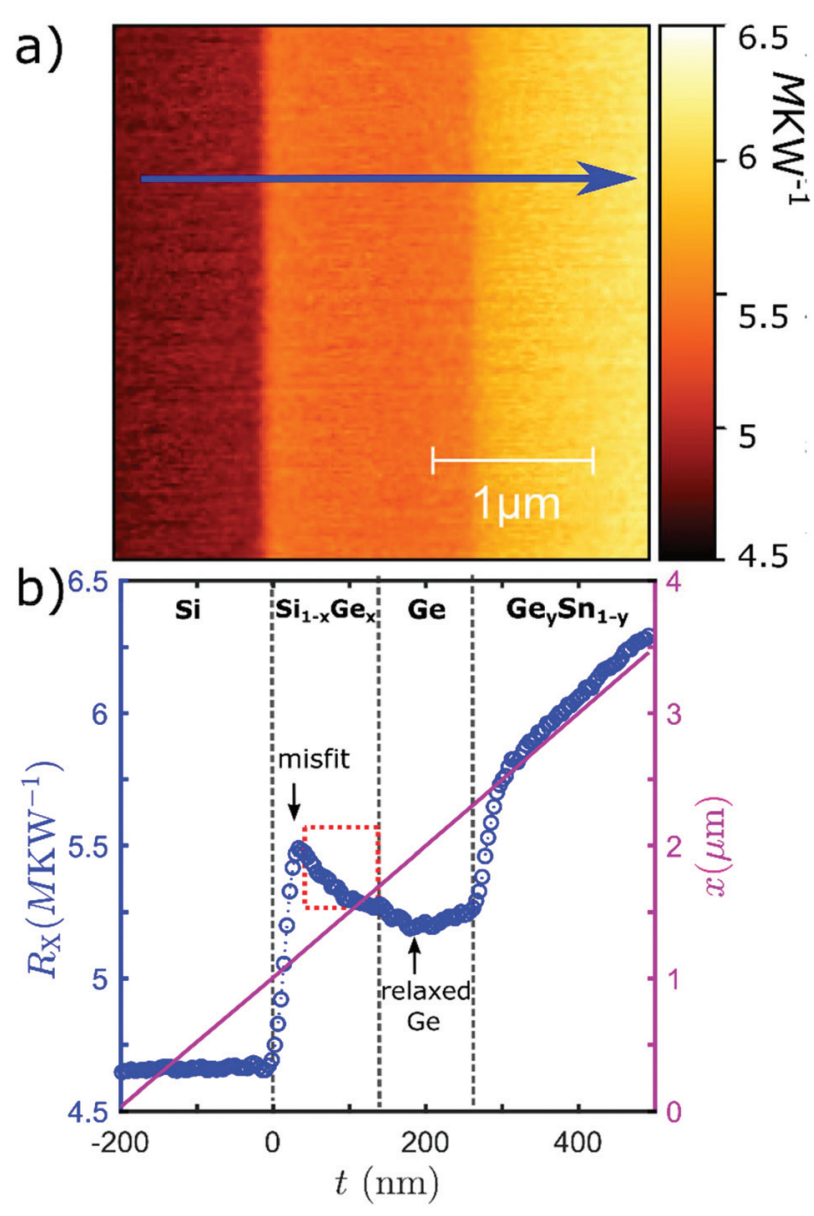

Fig. 2 (a) Thermal contact resistance $R_{X}$ image across the cross-sectioned $\mathrm{Si} / \mathrm{Ge}_{x} \mathrm{Si}_{1-x} / \mathrm{Ge}_{y} \mathrm{Sn}_{1-y}$ layers. Image is a zoom in the area shown in Fig. 1b. (b) $R_{\mathrm{X}}$ profile acquired from the image (blue, left axis), as indicated by the blue arrow by averaging 100 lines, and topography profile, $x$, (magenta, right axis) as a function of height, $t$, across $\mathrm{Si} / \mathrm{Ge}_{x} \mathrm{Si}_{1-x} /$ $\mathrm{Ge}_{y} \mathrm{Sn}_{1-y}$ layers. The areas of different materials are shown and are aligned with the image. The red dotted line denotes an area, where dislocation density reduces moving away from the interface. 
concentration. We excluded the possibility that the thermal resistance variations had their origin at tip-sample contact area variations since we did not observe any significant topography variations in the cross-sectioned area (see ESI note $7 \dagger$ for relevant profiles).

The most remarkable observation is that the resistance in the $\mathrm{Ge}_{x} \mathrm{Si}_{1-x}$ decreased in the middle region (red dotted area in Fig. 2), which is consistent with the thermal conductivity increase of $\mathrm{Ge}_{x} \mathrm{Si}_{1-x}$ with the decrease in the Si content. ${ }^{30}$ This significant continuing drop in the thermal resistance extending to $200 \mathrm{~nm}$ thickness can be linked with the reduction of the dislocation density as one moves away from the interface. According to second ion mass spectroscopy (SIMS) measurements (see ESI note $6 \dagger$ ), the Ge content of the $\mathrm{Ge}_{x} \mathrm{Si}_{1-x}$ layer quickly increased from 0 to $80 \%$ in the first $\sim 25 \mathrm{~nm}$. This region corresponds to the sharp increase measured in the thermal resistance. Beyond the low thermal conductivity of $\mathrm{Ge}_{x} \mathrm{Si}_{1-x}$ alloys with more than $10 \%$ Ge (compared to the pure silicon one), ${ }^{30}$ such drastic changes in the layer composition were also likely to induce phonon scattering processes, and created a low thermal conductivity, which translated into a high thermal resistance increase. Then, between 25 and $125 \mathrm{~nm}$, the Ge content increased slowly from $80 \%$ to $90 \%$ in the remaining of the layer, where the thermal resistance decrease is observed. The thermal conductivity of the $\mathrm{Ge}_{x} \mathrm{Si}_{1-x}$ alloys increases with the Ge content above $80 \%$ Ge. We can then attribute the thermal resistance decrease to this increasing thermal conductivity with thickness. In the Ge only layer, our measurement affords an even lower resistance. This can also be linked to the Ge content, which reaches $100 \%$ in this region (see ESI note $6 \dagger$ ), and thus provides a higher thermal conductivity.

We then investigated two $\mathrm{SiGe}_{x} / \mathrm{GeSn}_{y}$ samples that have similar composition, but different processing conditions, which are known to change the metastable GeSn alloy ${ }^{26-28}$ composition and crystallinity upon annealing at high temperatures. $^{31}$ Sn mobility inside the Ge can also increase drastically with temperature with $\mathrm{Sn}$ atoms tending to form clusters and segregates. $^{28}$ To assess these effects, we compared two samples: an as grown sample, and a sample that was subsequently annealed at $500{ }^{\circ} \mathrm{C}$ prior to the $\mathrm{xSThM}$ characterization. Fig. 3 shows the thermal resistance for these two samples. For comparison purposes, we normalized the signals to both the Si and Ge layers, which should not change due to the annealing process. Here, we observed almost no difference in the thermal transport in the $\mathrm{Si}_{1-x} \mathrm{Ge}_{x}$ region between the asgrown and annealed sample, which would be expected, as the annealing temperatures were well below ones needed to anneal the SiGe structures. In the pure Ge region, we obtained an almost flat response, which indicated that the spreading resistance was not affected by the increase of Ge layer thickness. When entering to the $\mathrm{Ge}_{0.9} \mathrm{Sn}_{0.1}$ layer, the resistance increased for both samples. This could be expected due to the lower thermal conductivity of GeSn alloys ${ }^{32,33}$ (between 1 and $10 \mathrm{~W}$ $\left.\mathrm{m}^{-1} \mathrm{~K}^{-1}\right)$ compared to pure $\mathrm{Ge}\left(\sim 20 \mathrm{~W} \mathrm{~m}^{-1} \mathrm{~K}^{-1}\right.$ for $100 \mathrm{~nm}$ film $\left.{ }^{32,34}\right)$. However, a notable difference was observed between

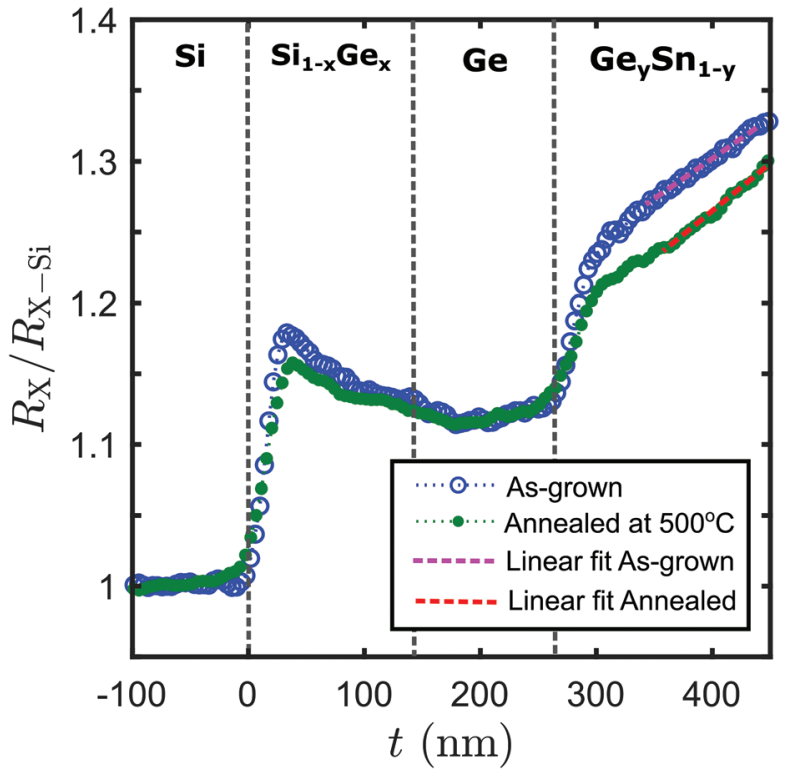

Fig. 3 Thermal resistance (normalized with Si and Ge layer thermal resistance) profile as obtained by averaging 100 lines as a function of height for the as-grown and annealed samples. With dotted lines, the fitted curves of the $\mathrm{Ge}_{y} \mathrm{Sn}_{1-y}$ region are indicated. For the as-grown sample, the slope is $\mathrm{d} y / \mathrm{d} x \approx 5.8 \pm 0.2 \times 10^{-4} \mathrm{~nm}^{-1}$, and for the annealed, it is $\mathrm{d} y / \mathrm{d} x \approx 6.9 \pm 0.2 \times 10^{-4} \mathrm{~nm}^{-1}$

the as-grown and the annealed samples by the analysis of the absolute value and the derivative of the thermal resistance in these layers. The lower absolute value suggests a higher concentration of Ge near the interface. Simultaneously, the higher derivative for the annealed sample suggests, similarly to the $\mathrm{Si}_{1-x} \mathrm{Ge}_{x}$ region, a different GeSn crystal quality. Annealing is likely to create clusters of Sn inside the Ge, which acted as phonon scattering elements, hence reducing the thermal conductivity.

For this complex nanostructure, xSThM here allowed for the first time to directly link the variation of the local thermal conductance due to the layer composition, crystalline defects and the precipitate nanostructuring, via the physical properties of buried layers, which would be impossible to access otherwise. The next section addresses the vital question of how to use xSThM for the quantitative thermal measurements in such layers.

\section{Quantitative measurements of thermal conductivity and interfacial thermal resistance}

Having established the high performance of thermal transport mapping in 3D layers, we first used xSThM to quantitatively deduce the thermal properties of relatively simple $3 \mathrm{D}$ structures comprised of isotropic layers. The wedge-like cut enables SThM measurements as function of material thickness that changes depending on the position of the probe across the cut (see Fig. 4a). As the tip-surface and wedge sample - substrate thermal resistance are independent of the tip position, using 


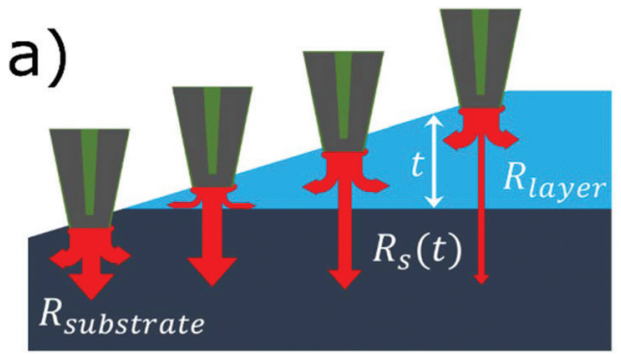

b)
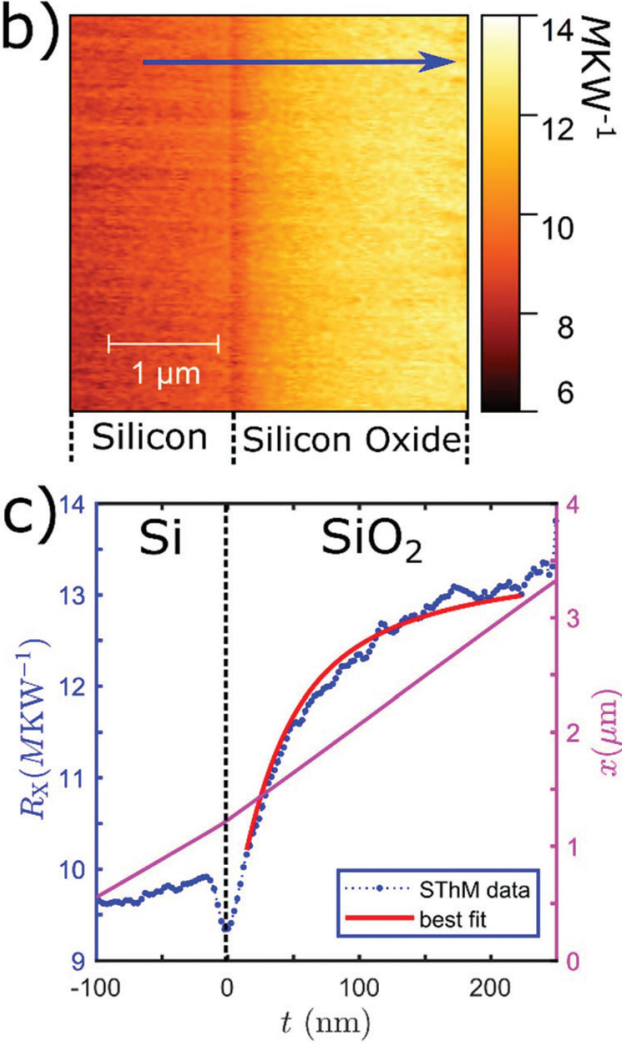

Fig. 4 (a) Schematic view of the XSThM scanning along the polished sample with an increase in the thickness. The arrows show the heat flow direction, with their width denoting the increased heat flow. At the limit of a thick layer, heat flow is mostly lateral within the layer. The top surface was removed from the image because it is not nano-sectioned, its roughness is very different from the top surface one, and also the top surface can be contaminated during the sectioning due to re-deposition of material, and these measurements should not be compared directly with the cross-sectioned area in this case (see ESI Note $8 \dagger$ for the thermal image including the top surface). (b) Thermal contact resistance, $R_{x}$, map of the $300 \mathrm{~nm} \mathrm{SiO}$ on $\mathrm{Si}$ cross-sectioned sample. (c) $R_{x}$ profile acquired from the XSThM image by averaging 100 lines as a function of height across $\mathrm{Si} / \mathrm{SiO}_{2}$ layers in the direction shown by the blue arrow at (b).

samples of varied thicknesses therefore allowed to separate the contribution of the interfacial thermal resistance and sample thermal conductivity in order to deduce the quantitative properties. $^{35-39}$

For the quantification of the thermal properties, we expressed $R_{\mathrm{X}}$ as a sum of two main components connected in series: the total contact thermal resistance between the probe and the sample, $R_{\mathrm{c}}$, and the total thermal spreading resistance within the sample, $R_{\mathrm{S}}$,

$$
R_{\mathrm{X}}=R_{\mathrm{c}}+R_{\mathrm{S}}
$$

In vacuum, $R_{\mathrm{c}}$ includes solid-solid contact thermal resistance, and in ambient environment, also water meniscus conductance. ${ }^{40}$ For the quantitative evaluation of the sample thermal resistance, we treated $R_{\mathrm{c}}$ as an effective probe-sample interface resistance dependent on the contact area and the sample thermophysical properties, and independent of $R_{\mathrm{s}}$. The SThM tip - sample contact area can be approximated ${ }^{7}$ by a disk of radius $a$, reflecting the solid-solid contact dimensions, and when in ambient conditions, may increase due to effects of water meniscus, providing an effective radius of the thermal contact.

The thermal spreading resistance depends on the nanoscale structure and the material composition of the sample. In the simple case of a bulk isotropic material and a contact radius above the phonon mean free path $\Lambda$, the thermal spreading resistance is given by:

$$
R_{\mathrm{S}}=\frac{1}{4 k a},
$$

where $k$ is the thermal conductivity of the bulk material. In case of the contact radius is much smaller than mean free path, the ballistic approximation can be used. ${ }^{41}$ Diffusive transport assumption remains valid if the heat source dimension $\alpha$ is bigger than $\Lambda$, indicating that the system Knudsen number $(K n=\Lambda / a)$ is smaller than 1 . In our system, $\Lambda$ was greatly reduced compared to the bulk values due to interface scattering and impurities. ${ }^{30}$ Except for dislocation-free $\mathrm{Si}$, where $\Lambda \sim 300 \mathrm{~nm},{ }^{42} \Lambda$ values are usually smaller than $50 \mathrm{~nm}$, the typical effective contact radius. ${ }^{32}$ In either case, if the contact dimensions or material did not change, the thermal resistance remained constant.

The basic element of any 3D nanostructure is a layer with thermal conductivity $k_{1}$ on the uniform substrate with thermal conductivity, $k_{\mathrm{s}}$. The angle wedge cut through the layer and the substrate, produced by BEXP nano-cross-sectioning, allowed us to approximate each measurement point as a layer of variable thickness. We then could use an isotropic model for $R_{\mathrm{s}}$ for the heat spreading within the layer on a substrate as described elsewhere: ${ }^{43,44}$

$$
R_{\mathrm{S}}(t)=\frac{1}{\pi k_{1} a} \int_{0}^{\infty}\left[\frac{1+K \exp \left(-\frac{2 \xi t_{\text {eff }}}{a}\right)}{1-K \exp \left(-\frac{2 \xi t_{\text {eff }}}{a}\right)}\right] J_{1}(\xi) \sin (\xi) \frac{\mathrm{d} \xi}{\xi^{2}},
$$

where $t_{\text {eff }}=t+r_{\text {int }} \times k_{1}$ is the effective thickness that depends on the interfacial resistance between the layer and the substrate per unit area, $r_{\text {int }}$, and the layer thickness, $t, J_{1}$ is the first Bessel function of the first kind, $\xi$ is an integration factor and where $K$ is defined as $K=\left(1-k_{\mathrm{s}} / k_{1}\right) /\left(1+k_{\mathrm{s}} / k_{1}\right)$. Note that this model can be extended to the orthotropic system ${ }^{45}$ to describe thermally anisotropic materials. This thickness varying 
thermal spreading resistance is connected with the variation in the direction of heat flow as layer thickness crosses from $t$ $\ll a$ to $t \gg a$, as schematically represented in Fig. 4a. At the limit of a thick layer with $t \gg a$, the spreading resistance is described by eqn (2).

We investigated three films of standard materials currently widely used in the semiconductor industry ${ }^{46,47}$ with potential for the next generation extreme UV (eUV) lithography: $60 \mathrm{~nm}$ spin-on carbon, $10 \mathrm{~nm}$ spin-on glass, complemented by the $300 \mathrm{~nm}$ thermally grown $\mathrm{SiO}_{2}$ on a $\mathrm{Si}$ substrate. In a BEXP section, thickness of the layer linearly varied with the position, and could be precisely quantified via topography. ${ }^{21}$ Most significantly, owing to the perfect near-atomic flatness of the cut, the tip-sample thermal resistance as well as the layer-substrate interfacial thermal resistance were constant, and did not depend on the layer thickness. This allowed us to perform direct fitting of the $R_{\mathrm{X}}$ vs. $t$ dependence using eqn (3), and therefore independently determine $k_{1}$ and $r_{\text {int }}$.

As expected, Fig. $4 \mathrm{~b}$ shows the $\mathrm{xSThM} R_{\mathrm{X}}$ map of a $300 \mathrm{~nm}$ thermal oxide on $\mathrm{Si}$ with thermal resistance of $\mathrm{Si}$ area lower than that of $\mathrm{SiO}_{2} \cdot{ }^{48}$ In Fig. 4c, topography and thermal resistance profiles taken along the blue arrow in Fig. $4 \mathrm{~b}$ are shown. The thermal resistance of $\mathrm{Si}$ is almost stable, while for $\mathrm{SiO}_{2}$, we observed a clear increase with an increase in the thickness corresponding to an increasing spreading resistance. A narrow dip at the $\mathrm{Si}-\mathrm{SiO}_{2}$ interface is attributed to the topographical variations at the interface that locally changes the contact area between the tip apex and the surface. These can occur at the junctions of the very dissimilar materials, but are not present as we can see in the uniform or smooth gradient materials. These topographical changes can be readily observed and eliminated from the measurements, or compensated by special algorithms. ${ }^{49}$ Note that the difference of Si thermal resistance with the sample presented in Fig. 2 is due to the different tip apex radii of the probe used. When the probe was solely in contact with the oxide layer, we can assume that the total tip-surface contact resistance $R_{\mathrm{c}}$ of eqn (1) is constant as material and contact area were not varied. We then applied the analytical model of eqn (3) using unknown parameters $R_{\mathrm{c}}, a$, $k_{1}$ and $r_{\text {int }}$ as fitting parameters (see Table 1 for the fitting results). In order to further reduce the number of fitting parameters, and thus increase the accuracy of the fit, we can analytically remove the $R_{\mathrm{c}}$ contribution by defining a new fitting function $f\left(t-t_{0}\right)=R_{\mathrm{S}}(t)-R_{\mathrm{S}}\left(t_{0}\right)$. However, $R_{\mathrm{c}}$ could be obtained

Table 1 Parameters obtained by fitting eqn (1) to experimental data. For $\mathrm{SiO}_{2}$, literature values are used to obtain a and $r_{\text {int }}$. Grey shaded cells are assumed values with contact radius for the probe for spin-on carbon and spin-on glass taken from the SiOx calibration measurements

\begin{tabular}{llll}
\hline $\begin{array}{l}\text { Fitting } \\
\text { parameters }\end{array}$ & $\begin{array}{l}300 \mathrm{~nm} \\
\text { thermal SiOx }\end{array}$ & $\begin{array}{l}60 \mathrm{~nm} \text { spin-on } \\
\text { carbon }\end{array}$ & $\begin{array}{l}10 \mathrm{~nm} \text { spin-on } \\
\text { glass }\end{array}$ \\
\hline$k_{1}\left(\mathrm{~W} \mathrm{~m}^{-1} \mathrm{~K}^{-1}\right)$ & 1.0 & $0.8 \pm 0.1$ & $0.3 \pm 0.1$ \\
$r_{\mathrm{int}}\left(10^{-9} \times \mathrm{K} \mathrm{m}^{2} \mathrm{~W}^{-1}\right)$ & 1.0 & $4 \pm 2$ & $2 \pm 2$ \\
$R_{\mathrm{c}}\left(10^{6} \times \mathrm{K} \mathrm{W}^{-1}\right)$ & $9.0 \pm 0.1$ & $5.3 \pm 0.1$ & $6.0 \pm 0.2$ \\
$a(\mathrm{~nm})$ & 56 & 56 & 56
\end{tabular}

afterwards by simply finding the offset to match the measured resistance (see ESI $\dagger$ for more details). The independent determination of several independent thermal parameters in a single experiment became possible, as the measurements were performed for the varied thickness of the sample, which was equivalent to the multiple experiments on the same system. ${ }^{39}$ This approach is effective if we assume that the layer thickness does not affect its thermal conductivity, and that layer thermal conductivity is isotropic (see ESI $\uparrow$ for more details).

In addition, we could use the $300 \mathrm{~nm}$ oxide sample as a proxy to an "infinitely thick" calibration sample, allowing to determine the contact radius $a$. By assuming literature values for the $\mathrm{SiO}_{2}$ thermal conductivity $\left(k_{\mathrm{SiO} 2} \approx 1 \mathrm{~W} \mathrm{~m}^{-1} \mathrm{~K}^{-1}\right)^{50,51}$ and the interfacial thermal resistance between silicon oxide and silicon $\left(r_{\text {int }_{\mathrm{Si}_{-} \mathrm{SiO}_{2}}} \approx 1 \times 10^{-9} \mathrm{~K} \mathrm{~m}^{2} \mathrm{~W}^{-1}\right),{ }^{50,52}$ this value only weakly affects the fitting result given the large thickness of the calibration layer) the only fitting parameter was $a$. We obtained $a=56 \mathrm{~nm}$, which is reasonable for the SThM probe used, with a good fit quality (see Fig. 4b). It should be noted that all three samples were thermally imaged sequentially under same geometrical settings and the same SThM probe, and therefore, no significant change in a was expected from sample to sample.

As eqn (3) is valid for all values of layer thickness, the power of our method also relies on its ability to measure very thin layers. By effectively expanding the thickness scale by 5 times, and hence the thickness resolution, it allowed us to study the physical properties of nanoscale layers that were only few $\mathrm{nm}$ thick. Such thin layers were impossible to be addressed by vertical cross-section due to the SThM tip diameter. ${ }^{21}$ Applying the same method, and using the calibrated effective contact radius, we measured thermal conductivities and the interfacial resistances of $60 \mathrm{~nm}$ spin-on carbon and $10 \mathrm{~nm}$ spin-on glass (see Table 1). We noted that a general agreement of a trend between the values was obtained, as spin-on carbon was expected to be more thermally conductive than spin-on glass. Experimental data and fitted curves are available in the ESI (Note $4 \dagger$ ).

Finally, it was also possible to quantify thermal conductance anisotropy of more complicated gradient structures with the use of FEA. In this context, we studied the $\mathrm{Si}_{x} \mathrm{Ge}_{1-x}$ gradient material, a good candidate for high temperature thermoelectrics. We found that as the SThM probe scanned across different layers of increased Ge concentration, the thermal resistance at the tip apex increased in good agreement with previous studies on $\mathrm{Si}_{1-x} \mathrm{Ge}_{x}$ alloys. ${ }^{30}$ Modelling the $\mathrm{Si}_{x} \mathrm{Ge}_{1-x}$ by FEA enabled us to reproduce experimentally acquired thermal resistance as a function of the Ge concentration (see ESI Supporting Note $2 \uparrow$ for details).

\section{Conclusions}

Combining an SPM-compatible nano-cross-sectional tool with SThM, we were able to map and measure with nanoscale resolution the thermal conductivity and interfacial thermal resistance of buried layers and interfaces in complex gradient 
compound semiconductor nanostructures, which were not accessible previously. We applied a new approach to the investigation of the thermal conductance of nanomaterials, providing the depth profiling of thermophysical properties with a depth resolution below $10 \mathrm{~nm}$. We have directly measured heat transport in nano-layered anisotropic systems, such as potential optoelectronic $\mathrm{Si} / \mathrm{Si}_{x} \mathrm{Ge}_{1-x} / \mathrm{Ge} / \mathrm{Ge}_{y} \mathrm{Sn}_{1-y}$ and thermoelectric $\mathrm{Si}_{x} \mathrm{Ge}_{1-x}$ materials, showing an excellent match with the theoretical data with no fitting parameters, and observing composition variations and dislocation-impeded thermal transport in nanoscale thin layers. Furthermore, using a complimentary modelling approach, we could deduce the quantitative values of thermal conductivity in microelectronic thin films and molecular beam epitaxy layers for the next generation optoelectronics and thermoelectrics. Our study demonstrates the ability to differentiate between thermal conductivity and interfacial thermal resistance in these samples, and to explore local stoichiometry and crystalline defects in nanostructured materials and devices. This approach could prove to be vitally important for quantitative nanoscale thermal characterization aspects that are currently largely missing in nanomanufacturing.

\section{Author contributions}

O.K., J.S. and B.R. conceived the idea of the approach and its application. J.S. performed the measurements, data analysis and modelling. C.E. performed the vacuum experiments. L.H. and A.R. prepared the sample with BEXP, and helped with the experiments. L.H., M.O. and J.S. grew the SiGeSn samples, and provided insights on the sample structure and behavior. M.A. and M.G. prepared the SiGe samples. A.S., F.A., and C.S. provided the samples and interpretation of the thermal properties of SiGe. O.K., J.S., E.C provided interpretation of the experimental data and theoretical analysis. J.S. drafted the manuscript, O.K., C.E. revised it, and all authors contributed to its final version.

\section{Conflicts of interest}

There are no conflicts to declare.

\section{Acknowledgements}

The authors acknowledge the EU QUANTIHEAT FP7 project no. 604668 and the Horizon 2020 Graphene Flagship Core 3 project no. 881603, EPSRC EP/G015570/1 EP/K023373/1, EP/ G06556X/1. EP/V00767X/1 and EP/P006973/1 and Faraday Institution NEXGENNA project for the overall support, and Paul Instrument Fund, c/o The Royal Society grant on "Infrared non-contact atomic force microscopy (ncAFM-IR)" for the equipment support. We are also grateful to our industrial collaborators Leica Microsystems, Lancaster Materials Analysis Ltd and Bruker for the financial and instrumentation support. LTD to ICN2 is supported by the Spanish MINECO (Severo Ochoa Centers of Excellence Program under Grant SEV-2017-0706 and CEX2019-000917-S) and by the Generalitat de Catalunya (Grants 2017SGR806, 2017SGR488, and the CERCA Program). We thank Severine Gomès from CNRS and Harry Hoster from Lancaster Energy for the helpful discussions on the measurements, and Andy Cockburn and Mike Kocsic from IMEC for the interesting materials and discussion of applications.

\section{Notes and references}

1 C. A. Mack, IEEE Trans. Semicond. Manuf., 2011, 24, 202207.

2 A. Majumdar, Nat. Nano., 2009, 4, 214-215.

3 S. W. Fong, C. M. Neumann and H. P. Wong, IEEE Trans. Electron Devices, 2017, 64, 4374-4385.

4 S. Volz, Microscale and nanoscale heat transfer, Springer, 2007.

5 H. J. Jang, C. R. Ryder, J. D. Wood, M. C. Hersam and D. G. Cahill, Adv. Mater., 2017, 29, 1700650.

6 W. Jeong, S. Hur, E. Meyhofer and P. Reddy, Nanoscale Microscale Thermophys. Eng., 2015, 19, 279-302.

7 S. Gomès, A. Assy and P.-O. Chapuis, Phys. Status Solidi A, 2015, 212, 477-494.

8 M. E. Pumarol, M. C. Rosamond, P. Tovee, M. C. Petty, D. A. Zeze, V. Falko and O. V. Kolosov, Nano Lett., 2012, 12, 2906-2911.

9 L. Shi and A. Majumdar, J. Heat Transfer, 2002, 124, 329337.

10 V. V. Gorbunov, N. Fuchigami, J. L. Hazel and V. V. Tsukruk, Langmuir, 1999, 15, 8340-8343.

11 K. Luo, R. W. Herrick, A. Majumdar and P. Petroff, Appl. Phys. Lett., 1997, 71, 1604-1606.

12 T. Lee, X. Guo, G. Shen, Y. Ji, G. Wang, J. Du, X. Wang, G. Gao, A. Altes and L. Balk, Microelectron. Reliab., 2002, 42, 1711-1714.

13 K. W. Park, E. M. Krivoy, H. P. Nair, S. R. Bank and E. T. Yu, Nanotechnology, 2015, 26, 265701.

14 J. Juszczyk, M. Krzywiecki, R. Kruszka and J. Bodzenta, Ultramicroscopy, 2013, 135, 95-98.

15 E. Jung, G. Hwang, J. Chung, O. Kwon, J. Han, Y.-T. Moon and T.-Y. Seong, Appl. Phys. Lett., 2015, 106, 041114.

16 D. Choi, N. Poudel, S. Park, D. Akinwande, S. B. Cronin, K. Watanabe, T. Taniguchi, Z. Yao and L. Shi, ACS Appl. Mater. Interfaces, 2018, 10, 11101-11107.

17 J. L. Bosse, I. Grishin, B. D. Huey and O. V. Kolosov, Appl. Surf. Sci., 2014, 314, 151-157.

18 O. V. Kolosov, I. Grishin and R. Jones, Nanotechnology, 2011, 22, 185702.

19 O. V. Kolosov and I. Grishin, US Patent 9,082,587, 2015.

20 A. J. Robson, I. Grishin, R. J. Young, A. M. Sanchez, O. V. Kolosov and M. Hayne, ACS Appl. Mater. Interfaces, 2013, 5, 3241-3245. 
21 A. J. Robson, I. Grishin, R. J. Young, A. M. Sanchez, O. V. Kolosov and M. Hayne, ACS Appl. Mater. Interfaces, 2013, 5, 3241-3245.

22 P. S. Dobson, G. Mills and J. M. R. Weaver, Rev. Sci. Instrum., 2005, 76, 054901.

23 P. Tovee, M. E. Pumarol, D. A. Zeze, K. Kjoller and O. Kolosov, J. Appl. Phys., 2012, 112, 114317.

24 C. Evangeli, J. Spiece, S. Sangtarash, A. J. Molina-Mendoza, M. Mucientes, T. Mueller, C. Lambert, H. Sadeghi and O. Kolosov, Adv. Electron. Mater., 2019, 5, 1900331.

25 J. Spiece, C. Evangeli, K. Lulla, A. Robson, B. Robinson and O. Kolosov, J. Appl. Phys., 2018, 124, 015101.

26 N. Bhargava, J. P. Gupta, N. Faleev, L. Wielunski and J. Kolodzey, J. Electron. Mater., 2017, 46, 1620-1627.

27 W. Wang, L. Li, Q. Zhou, J. Pan, Z. Zhang, E. S. Tok and Y.-C. Yeo, Appl. Surf. Sci., 2014, 321, 240-244.

28 L. Kormoš, M. Kratzer, K. Kostecki, M. Oehme, T. Šikola, E. Kasper, J. Schulze and C. Teichert, Surf. Interface Anal., 2017, 49, 297-302.

29 M. Oehme, D. Buca, K. Kostecki, S. Wirths, B. Holländer, E. Kasper and J. Schulze, J. Cryst. Growth, 2013, 384, 71-76.

30 A. Iskandar, A. Abou-Khalil, M. Kazan, W. Kassem and S. Volz, J. Appl. Phys., 2015, 117, 125102.

31 H. Mahmodi and M. R. Hashim, Mater. Res. Express, 2016, 3, 106403.

32 S. N. Khatami and Z. Aksamija, Phys. Rev. Appl., 2016, 6, 014015.

33 N. Uchida, T. Maeda, R. R. Lieten, S. Okajima, Y. Ohishi, R. Takase, M. Ishimaru and J.-P. Locquet, Appl. Phys. Lett., 2015, 107, 232105.

34 R. Cheaito, J. C. Duda, T. E. Beechem, K. Hattar, J. F. Ihlefeld, D. L. Medlin, M. A. Rodriguez, M. J. Campion, E. S. Piekos and P. E. Hopkins, Phys. Rev. Lett., 2012, 109, 195901.

35 A. Makris, T. Haeger, R. Heiderhoff and T. Riedl, RSC Adv., 2016, 6, 94193-94199.
36 R. Heiderhoff, H. Li and T. Riedl, Microelectron. Reliab., 2013, 53, 1413-1417.

37 S. Gome, L. David, V. Lysenko, A. Descamps, T. Nychyporuk and M. Raynaud, J. Phys. D Appl. Phys., 2007, 40, 66776683.

38 J. Juszczyk, A. Kazmierczak-Balata, P. Firek and J. Bodzenta, Ultramicroscopy, 2017, 175, 81-86.

39 J. L. Bosse, M. Timofeeva, P. D. Tovee, B. J. Robinson, B. D. Huey and O. V. Kolosov, J. Appl. Phys., 2014, 116, 134904.

40 A. Assy and S. Gomes, Nanotechnology, 2015, 26, 355401.

41 R. Prasher, Nano Lett., 2005, 5, 2155-2159.

42 K. T. Regner, D. P. Sellan, Z. Su, C. H. Amon, A. J. H. McGaughey and J. A. Malen, Nat. Commun., 2013, 4, 1640.

43 Y. S. Muzychka, M. M. Yovanovich and J. R. Culham, J. Thermophys. Heat Transfer, 2004, 18, 45-51.

44 Y. S. Muzychka, J. Thermophys. Heat Transfer, 2014, 28, 313319.

45 F. Menges, H. Riel, A. Stemmer, C. Dimitrakopoulos and B. Gotsmann, Phys. Rev. Lett., 2013, 111, 205901.

46 A. Frommhold, R. E. Palmer and A. P. Robinson, J. Micro/ Nanolithogr., MEMS, MOEMS, 2013, 12, 033003-033003.

47 A. Frommhold, J. Manyam, R. E. Palmer and A. P. G. Robinson, Microelectron. Eng., 2012, 98, 552-555.

48 B. Deng, A. Chernatynskiy, M. Khafizov, D. H. Hurley and S. R. Phillpot, J. Appl. Phys., 2014, 115, 084910.

49 P. Klapetek, J. Martinek, P. Grolich, M. Valtr and N. J. Kaur, Int. J. Heat Mass Transfer, 2017, 108, 841-850.

50 A. Al Mohtar, G. Tessier, R. Ritasalo, M. Matvejeff, J. Stormonth-Darling, P. Dobson, P. Chapuis, S. Gomès and J. Roger, Thin Solid Films, 2017, 642, 157-162.

51 K. Goodson, M. Flik, L. Su and D. Antoniadis, J. Heat Transfer, 1994, 116, 317-324.

52 J. Chen, G. Zhang and B. Li, J. Appl. Phys., 2012, 112, 064319. 